\title{
Now is the time to consider measures against next wave of COVID-19
}

\section{Jong-Koo Lee}

Department of Family Medicine, Seoul National University College of Medicine, Seoul, Korea

Received: December 17, 2021

Accepted: December 20, 2021

Corresponding author:

Jong-Koo Lee

Department of Family

Medicine, Seoul National

University College of Medicine,

103 Daehak-ro, Jongno-gu,

Seoul 03080, Korea

E-mail: docmohw@snu.ac.kr
The Korean government has reported an average of 6,000 coronavirus disease 2019 (COVID-19) cases daily, and the case fatality rate has increased to $0.83 \%$ this week [1]. Since the complete vaccination rate exceeded $75 \%$ one and a half months ago, the government showed confidence in switching to the "with corona" policy, but according to the latest government data, this week's average incidence rate per day is 11.7 cases per 100,000 people, despite the high vaccination completion rate (81.1\%) [2].

This assumption that herd immunity could allow society to return to normal in November was unfortunately erroneous. Initially, it was estimated that the number of patients would increase slightly after implementing the "with corona" policy, but it is considered somewhat surprising that the number of patients with severe disease and mortality increased. Therefore, we are faced with the following questions: what is the current situation, and what are the urgent short-term and mid-to-long-term measures to reduce the number of deaths? Since it appears that COVID-19, which has transformed to delta, Omicron variants and has spread worldwide, cannot be managed by vaccination alone, it is now time to refine countermeasures against COVID-19 as an endemic disease.

First, in the short term, it is necessary to quickly increase the booster vaccination rate for the elderly and at-risk groups. Starting at the end of February 2021, the following groups were prioritized for vaccinations: health workers responsible for infection control at health centers, doctors and nurses at hospitals, residents of nursing facilities, the elderly, and chronically ill individuals with underlying diseases. However, the antibody titers of these elderly and vulnerable groups fell faster than expected, resulting in more immune escape in this group and increasing the number of breakthrough infections. In order to strengthen the effects of boosters, the interval until booster doses has been reduced to 3 months. According to a report published on December 13, the infection prevention rate of vaccines is $57.6 \%$, and the booster vaccination rate for those eligible aged 60 and over is $31.4 \%$. These data mean that we cannot prevent this rapid outbreak without special measures. In addition, although the effects of vaccination on preventing serious illness and death remain $92.1 \%$ and $90.7 \%$, respectively, as the number of patients increases, the number of serious patients is also increasing. Therefore, the goal of a complete COVID-19 vaccination schedule should be revised to include all 3 doses. At the same time, in order to reduce the exposure of the elderly population to COVID-19, gatherings should be restricted for at least 1 month at the end of 2021 and the beginning of 2022, activities associated with trace-test-isolation- 
quarantine (TTIQ) must be reinforced by recruit of infection control team.

Second, strengthened efforts should be made to increase the vaccine uptake rate among vaccine-hesitant individuals and children aged 12 to 17 years. Ultimately, COVID-19 will have to be treated as a universally vaccinated disease, as it is endemic. In other words, vaccinations for endemic diseases, such as measles and seasonal influenza, are regularly administered for eradication. Therefore, people must be convinced of the necessity of vaccination by explaining the benefits and disadvantages of the currently used mRNA vaccines. For example, adverse events have been reported in about 4 out of 1,000 people, but $96 \%$ of adverse events are mild, and diseases associated with severe side effects are very rare.

Anaphylaxis has been reported in 572 cases, myocarditis in 300 cases (Pfizer 206 cases, Moderna 94 cases). Only 10 cases of myocarditis have been reported after the AstraZeneca vaccine [3]. In the United Kingdom, COVID-19 myocarditis has been reported to occur in 40 patients per 1 million without vaccination, but in about 10 cases when 2 doses of an mRNA vaccine are administered [4]. In other words, medical personnel need to provide more accurate explanations of the benefits of vaccines in order to increase the vaccination rate. Regarding mandatory vaccinations through the National Immunization Program, it is necessary to check individuals' vaccination history when they use public facilities, just like how vaccination is a requirement for admission at the time of school entrance. In other words, the current quarantine pass should be converted into a daily measure instead of being considered a temporary measure.

Third, the health care system should be strengthened. Instead of taking measures against COVID-19 as a pandemic, it should now be recognized that COVID-19 is an endemic disease that causes more than 500,000 cases and about 5,000 deaths every year. Therefore, COVID-19 should be managed as an endemic disease that leads to a similar death toll to the estimated 2,300 to 5,300 deaths due to influenza every year [5]. Our society has accepted the reality that about 10,000 people can die from respiratory infections, including influenza and COVID-19, each year. There is a potential demand for hospitalization of 500,000 to 1 million people every year, and it is necessary to prepare a medical care system that can treat 100,000 to 200,000 intensive care unit patients with $2 \%$ of aggravated cases among all ill patients. Of course, infection control for legally defined infectious diseases must be thoroughly implemented to prevent hospital-acquired infections, and treatment will be provided free of charge as it is today, but if appropriate preventive measures are not taken and mandatory vaccinations are not received, the government will not support the fees for care. Since most patients have mild cases, the diagnosis, treatment, prevention, and isolation system should be reorganized and centered on public health centers and district medical institutions so that primary care is provided for around $80 \%$ of patients. Infection control teams, home visiting health teams, telemedicine, designated evacuation medical institutions, night call centers, and patient transport systems must be created to reduce the demand for expensive hospitalization. Oral antiviral drugs will enable primary care physicians to care for patients, and will play a role in preventing infections and reducing the quarantine period.

Fourth, a preventive system must be established. Daily efforts to avoid infection are very important for a step-bystep recovery. In addition to washing hands, mask-wearing, and ventilating, it is necessary to use Information and Communications Technology (ICT) tools and apps that help check for fever when entering various facilities (regardless of vaccination status), prompt voluntary testing in case of doubt, and determine possible contacts with confirmed cases. Through these measures, trace-test-isolationquarantine (TTIQ) can improve performance and perfection restrictions on access can be minimized. In addition to efforts to improve vaccination at the local level, public health centers should activate a 'prevention committee system in the community' for infectious disease prevention and control by the law in order to report patients to local medical institutions, help conduct related epidemiological investigations, and provide patient education. They should be in charge of facility inspection and education to prevent cluster outbreaks. In addition, booster vaccination strategies such as ad hoc vaccination and TTIQ for epidemic management in case of a cluster outbreak will block transmission and reduce the side effects caused by social distancing.

\section{Notes}

\section{Ethics Approval}

Not applicable.

\section{Conflicts of Interest}

The author has no conflicts of interest to declare.

\section{Funding}

None.

\section{References}

1. Our World in Data. Case fatality of South Korea [Internet]. Our World in Data; 2021 [cited 2021 Dec 13]. Available from: https:// 
ourworldindata.org/covid-cases.

2. Korea Disease Control and Prevention Agency (KDCA). Weekly epidemiologic report, December 13, 2021 [Internet]. Cheongju: KDCA; 2021 [cited 2021 Dec 13]. Available from: https://www.kdca. go.kr/board/board.es? $\mathrm{mid}=$ a205010100008bid =00158list_no = $7179258 c$ c_code $=$ \&act $=$ view8nPage $=2$. Korean .

3. Korea Disease Control and Prevention Agency (KDCA). Weekly vaccine adverse event report, December 16, 2021 [Internet]. Cheongju: KDCA; 2021 [cited 2021 Dec 17]. Available from: https://ncv.kdca.go.kr/ board.es? $\mathrm{mid}=$ a117070100008bid $=0032$. Korean .
4. Patone M, Mei XW, Handunnetthi L, et al. Risks of myocarditis, pericarditis, and cardiac arrhythmias associated with COVID-19 vaccination or SARS-CoV-2 infection. Nat Med 2021 Dec 14 [Epub]. https://doi.org/10.1038/s41591-021-01630-0.

5. Park C, Park S, Lee G, et al. Estimation of excess mortality associated with influenzas in Korea. Public Health Wkly Rep 2021;14:1150-61. Korean. 